TRANSACTIONS OF THE

AMERICAN MATHEMATICAL SOCIETY

Volume 348, Number 9, September 1996

\title{
ON THE ORDERING OF $n$-MODAL CYCLES
}

\author{
CHRIS BERNHARDT
}

\begin{abstract}
The forcing relation on $n$-modal cycles is studied. If $\alpha$ is an $n$ modal cycle then the $n$-modal cycles with block structure that force $\alpha$ form a $2^{n}$-horseshoe above $\alpha$. If $n$-modal $\beta$ forces $\alpha$, and $\beta$ does not have a block structure over $\alpha$, then $\beta$ forces a 2-horseshoe of simple extensions of $\alpha$.
\end{abstract}

\section{INTRODUCTION}

Continuous maps of the interval induce a partial order on the set of cyclic permutations (cycles).

Suppose a map of the interval has a periodic orbit $P=\left\{p_{1}, \ldots, p_{n}\right\}$ labelled so that $p_{1}<\cdots<p_{n}$. Then $P$ has cycle type $\alpha$ if $\alpha$ is a cycle with the property that $f\left(p_{i}\right)=p_{j}$ if and only if $\alpha(i)=j$. A cycle $\alpha$ forces a cycle $\eta$, written $\alpha \geq \eta$, if every continuous map of the interval that has a periodic point of cycle type $\alpha$ also has a periodic point with cycle type $\eta$. Baldwin [2] showed that the forcing relation was a partial order on the set of cycles.

The structure of the set of cycles ordered by the forcing relation is complicated. The local structure is known: immediately above any cycle lie all doubles of that cycle (see [4]). A cycle $\alpha$ of period $n$ is a double if $n$ is even and for $k=1, \ldots, n / 2$, $\alpha(2 k-1)$ and $\alpha(2 k)$ are consecutive integers. (In this case, $\alpha$ is a "double" of the permutation of $\{1, \ldots, n / 2\}$ defined by $i \mapsto j$ if $\{\alpha(2 i-1), \alpha(2 i)\}=\{2 j-1,2 j\})$.

Let $\alpha$ and $\beta$ denote cycles. Suppose $\alpha$ has period $n$ and $\beta$ has period $m$. Then we say a cycle $\gamma$ has a block structure of type $\alpha$ by $\beta$, denoted $\alpha^{\beta}$, if $\gamma$ has period $n m$ and the properties that, for $1 \leq k \leq n, \gamma$ sends $\{(k-1) m+1,(k-1) m+2, \ldots, k m\}$ to $\{(\alpha(k)-1) m+1,(\alpha(k)-1) m+2, \ldots, \alpha(k) m\}$ and that, for at least one $k$, $\{(k-1) m+1,(k-1) m+2, \ldots, k m\}$ has cycle type $\beta$ under the $n$th iterate of $\gamma$. If $\gamma$ is monotonic on all but one of the sets $\{(k-1) m+1,(k-1) m+2, \ldots, k m\}$ then $\gamma$ will be called a simple extension of $\alpha$. (See [1] for many results concerning these ideas.)

Given $\beta$, a cyclic permutation of $\{1, \ldots, m\}$, let $L_{\beta}:[1, m] \rightarrow[1, m]$ denote the map defined by $L_{\beta}=\beta$ on $\{1, \ldots, m\}$ and $L_{\beta}$ is linear on each $[i, i+1]$. The nondegenerate intervals with respect to " $L_{\beta}$ is strictly monotone on $I$ " are called laps of $L_{\beta}$. The cycle $\beta$ is $n$-modal if $L_{\beta}$ has $n+1$ laps.

If attention is restricted to unimodal cycles then the ideas of block structure and simple extension coincide. The following result tells us more about the local ordering. This result follows from the work of Collet and Eckmann ([7], Theorem 11.2.6) after noting that the star product used in kneading theory corresponds to

Received by the editors April 3, 1995 and, in revised form, November 2, 1995.

1991 Mathematics Subject Classification. Primary 58F03; Secondary 58F20, 58F08.

(C)1996 American Mathematical Society 
the idea of simple extension (see also [5], Theorem 1). It is stated in terms of unimodal cycles with one maximum, but of course the analogous statement for unimodal cycles with one minimum is also true.

Theorem 1. Let $\alpha, \beta, \delta$ and $\eta$ denote unimodal cycles with one maximum. Then:

(1) There is a unique unimodal cycle of type $\alpha^{\beta}$.

(2) $\alpha^{\beta}$ forces $\alpha^{\delta}$ if and only if $\beta$ forces $\delta$.

(3) If $\eta$ forces $\alpha$ and $\eta$ does not have block structure over $\alpha$, then $\eta$ forces $\alpha^{\beta}$ for any unimodal $\beta$.

In what follows we shall see how these ideas generalize for $n$-modal cycles. It will be shown that if $\alpha$ is an $n$-modal cycle then the $n$-modal cycles with block structure that force $\alpha$ form a $2^{n}$-horseshoe above $\alpha$. If $n$-modal $\beta$ forces $\alpha$, and $\beta$ does not have a block structure over $\alpha$, then $\beta$ forces a 2 -horseshoe of simple extensions of $\alpha$.

\section{HORSESHOE MAPS}

An $n$-modal horseshoe map, denoted $H$, is defined as follows. $H:[0,1] \rightarrow[0,1]$ is continuous and $H$ maps each of the intervals $[(i-1) /(n+1), i /(n+1)]$ linearly onto $[0,1]$. There are two $n$-modal horseshoe maps, one with $H(0)=0$ and the other with $H(0)=1$. When we need to distinguish between these two types we will denote the first by $H^{+}$and the second by $H^{-}$.

Suppose that $P$ and $Q$ are two periodic orbits of $H$. The orbit $P$ is said to be tighter than the orbit $Q$ if

$$
\min _{q \in Q}\left|q-\frac{i}{n+1}\right| \leq \min _{p \in P}\left|p-\frac{i}{n+1}\right|, \quad i=1, \ldots, n
$$

The following two theorems come from [3].

Theorem 2. Let $\beta$ and $\alpha$ be two n-modal cycles. Then $\beta$ forces $\alpha$ if and only if there are representatives $Q$ of $\beta$ and $P$ of $\alpha$ in $H$ with $P$ tighter than $Q$.

Theorem 3. Let $\beta$ be an n-modal cycle. Then

(1) if $\beta$ is not a double, there is a representative of $\beta$ that is tighter than any other representative of $\beta$ in $H$.

(2) if $\beta$ is a double, there is a representative of $\beta / 2$ that is tighter than any representative of $\beta$ in $H$.

Suppose $P$ is a closed subset of $[0,1]$. For $i=1, \ldots, n+1$ (where $n$ is the modality of $H)$, define $d_{i}=\min \{|p-i /(n+1)|: p \in P\}$ and let $\bar{p}_{i}$ denote a point in $P$ such that $\left|\bar{p}_{i}-i /(n+1)\right|=d_{i}$. Then the P-truncation $H_{P}$ of $H$ is

$$
H_{P}(x)= \begin{cases}H\left(\bar{p}_{i}\right) & \text { if }|x-i /(n+1)| \leq d_{i}(i=0,1, \ldots, n), \\ H(x) & \text { otherwise. }\end{cases}
$$

The method for studying the local structure above a given cycle can be outlined as follows. First, find the tightest representative of the given cycle. Then construct the truncation of $H$ that corresponds to this representative. Then the maxima of this map will be "nudged" up and the minima "nudged" down. This process will create new periodic orbits. The cycles corresponding to these will be studied. However, first some more notation has to be introduced.

Various representatives of a given cycle will be described by their itineraries. Let $I_{i}$ denote the lap $[(i-1) /(n+1), i /(n+1)]$. Then the itinerary, It $(x)$, of a point 
$x \in[0,1]$, with respect to $H$, is a sequence $\tau=\{\tau(j)\}_{j=0}^{\infty}$ such that $H^{j}(x) \in I_{\tau(j)}$ for $j=0,1, \ldots$ There is a one-to-one correspondence between points in $[0,1]$ and their itineraries, except for turning points and their pre-images which have two itineraries. This ambiguity will be harmless, as we are interested in periodic points and the turning points are not periodic.

Itineraries are ordered in the following way. Define $\epsilon_{k}=+1$ (resp. -1) if $H$ is increasing (resp. decreasing) on $I_{k}, \zeta(0)=1$, and $\zeta(k)=\epsilon_{\tau(0)} \ldots \epsilon_{\tau(k-1)}$. If $\tau \neq \theta$ are two itineraries, let $k$ be the least integer such that $\tau(k) \neq \theta(k)$. Then $\tau<\theta$ if $\zeta(k)=+1$ and $\tau(k)<\theta(k)$, or if $\zeta(k)=-1$ and $\tau(k)>\theta(k)$. In the two other cases, $\tau>\theta$.

Lemma 4 (Milnor and Thurston [9], Lemma 3.1). Let $x$ and $y$ be points in $[0,1]$ having unique itineraries $\operatorname{It}(x)$ and $\operatorname{It}(y)$. Then $x<y$ if and only if $\operatorname{It}(x)<\operatorname{It}(y)$.

Itineraries come from the labelling of the laps of $H$. In what follows we shall often be looking at iterates of $H$. Clearly, $H^{j}$ is a horseshoe map with $(n+1)^{j}$ laps. The labelling of the laps on $H$ gives a natural labelling of the laps of $H^{j}$ as follows. Consider all possible itineraries of length $j$. There are $(n+1)^{j}$ of them. Order these itineraries as above, and let the $k^{t h}$ string be $\left(\tau_{k i}\right)_{i=1}^{j}$, which we shall denote $\tau_{k}^{j}$. Then the following lemma is easily seen to be true.

Lemma 5. If $x$ is in the $k^{\text {th }}$ lap of $H^{j}$, then the first $j$ terms of the itinerary of $x$, with respect to $H$, are $\tau_{k}^{j}$.

Since $\left(H^{j}\right)^{k}=H^{j k}$, it is clear that the itinerary of a point that comes from $H^{j}$, with the $k^{t h}$ lap labelled with $\tau_{k}^{j}$, is the same string of symbols as the itinerary of the point with respect to $H$.

Example 6. Consider the 2-modal cycle $\left(\begin{array}{llll}1 & 3 & 4 & 2\end{array}\right)$ and the associated horseshoe map, $H$, with three laps. One of the representatives of $\left(\begin{array}{llll}1 & 3 & 4 & 2\end{array}\right)$ has itinerary $12 \begin{array}{llllll}2 & 3 & 1 & 2 & 3 & 2\end{array}$.., which will be denoted $\left(\begin{array}{llll}1 & 2 & 3 & 2\end{array}\right)^{\infty}$.

Now consider $H^{2}$. It has 9 laps labelled from left to right by $13,12,11,21,22,23$, $33,32,31$. The itinerary of the given representative with respect to $H^{2}$ is $(1232)^{\infty}$, which is the same string of symbols as the itinerary above.

\section{ITINERARIES AND BLOCK STRUCTURE}

Proposition 7. Let $\alpha$ be an $n$-modal cycle. Let $H$ denote the associated $n$-modal horseshoe map. If $\alpha$ is not a double then there are $2^{n}$ representatives of $\alpha$ in $H$. If $\alpha$ is a double then there are $2^{n}-1$ representatives of $\alpha$ in $H$.

Proof. Let $x$ be the left point of an orbit in $H$ with cycle type $\alpha$. It is clear that if $L_{\alpha}^{i}(1)$ is not a critical point then it lies in a unique lap (call it the $k^{t h}$ ) of $L_{\alpha}$, and thus $H^{i}(x)$ must lie in the $k^{t h}$ lap of $H$. If $L_{\alpha}^{i}(1)$ is a critical point then it lies in two laps of $L_{\alpha}$, which gives two choices for the lap that $H^{i}(x)$ lies in. Since there are $n$ critical points we obtain $2^{n}$ possible itineraries for $x$. Denote this set of itineraries by $I(\alpha)$.

Suppose $\gamma \in I(\alpha)$. Suppose that $\alpha$ is a cycle of length $m$ and let $s$ denote the period of $\gamma$. Clearly, $s$ must divide $m$. Now $L_{\alpha}$ permutes the blocks $\{1, \ldots, s\}$, $\{s+1, \ldots, 2 s\}, \ldots,\{m-s+1, \ldots, m\}$ and is monotone on each block. Therefore $s=1$ or 2 . 
The map $\phi$ that sends $\{1, \ldots, m\}$ to the orbit of $x$, defined by $\phi\left(L_{\alpha}^{i}(1)\right)=$ $H^{i}(x)$, preserves the order $\geq$. The map that sends points to itineraries is also order preserving. So $\gamma$ must correspond to a representative of $\alpha$ or of $\alpha / 2$.

In [3] it is shown that if $\alpha$ is a double then at least one element of $I(\alpha)$ is a representative of $\alpha / 2$ - namely, the tightest. Given the construction of $I(\alpha)$, it is clear that at most one element of $I(\alpha)$ can be a representative of $\alpha / 2$.

The set $I(\alpha)$ of $2^{n}$ itineraries defined in the proof above will be called the itineraries of $\alpha$. The tightest itinerary of $\alpha$ will be the element of $I(\alpha)$ that corresponds to the tightest representative mentioned in the statement of Theorem 3 . (Note that if $\alpha$ is a double then the tightest itinerary of $\alpha$ is the itinerary of a representative of $\alpha / 2$.)

Given an itinerary $\tau=\left\{\tau_{k}\right\}_{k=0}^{\infty}$ we will let $\sigma$ denote the shift map that sends $\tau$ to $\sigma(\tau)=\left\{\tau_{k}\right\}_{k=1}^{\infty}$.

A basic result concerning shift maps is the following.

Lemma 8 (Milnor and Thurston [9]). It $(H(x))=\sigma(\operatorname{It}(x))$, for all $x \in[0,1]$.

We shall also use the notation $\left.\tau\right|_{n}$ to denote $\left\{\tau_{k}\right\}_{k=0}^{n-1}$, the first $n$ terms of $\tau$.

Lemma 9. Let $\alpha$ be an n-modal cycle of length $m$. Let $I(\alpha)=\left\{\theta_{1}, \ldots, \theta_{2^{n}}\right\}$. Let $\theta_{1}$ denote tightest itinerary of $\alpha$. Then

(1) $\left.\sigma^{i}\left(\theta_{1}\right)\right|_{m}, \ldots,\left.\sigma^{i}\left(\theta_{2^{n}}\right)\right|_{m}$ are all distinct.

(2) Either

$$
\left.\sigma^{i}\left(\theta_{1}\right)\right|_{m}=\min \left\{\left.\sigma^{i}\left(\theta_{1}\right)\right|_{m}, \ldots,\left.\sigma^{i}\left(\theta_{2^{n}}\right)\right|_{m}\right\}
$$

or

$$
\left.\sigma^{i}\left(\theta_{1}\right)\right|_{m}=\max \left\{\left.\sigma^{i}\left(\theta_{1}\right)\right|_{m}, \ldots,\left.\sigma^{i}\left(\theta_{2^{n}}\right)\right|_{m}\right\} .
$$

(3) The orientation of $\max \left\{\left.\sigma^{i}\left(\theta_{1}\right)\right|_{m}, \ldots,\left.\sigma^{i}\left(\theta_{2^{n}}\right)\right|_{m}\right\}$ is the opposite to the orientation of $\min \left\{\left.\sigma^{i}\left(\theta_{1}\right)\right|_{m}, \ldots,\left.\sigma^{i}\left(\theta_{2^{n}}\right)\right|_{m}\right\}$.

Proof. Statement (1) follows immediately from the construction of $I(\alpha)$. Statement (2) follows from Theorem 3. It remains to show the third statement.

Recall from the construction of $I(\alpha)$ that each of the truncated itineraries $\left.\sigma^{i}\left(\theta_{1}\right)\right|_{m}, \ldots,\left.\sigma^{i}\left(\theta_{2^{n}}\right)\right|_{m}$ is formed by changing up to $n$ of the terms of $\left.\sigma^{i}\left(\theta_{1}\right)\right|_{m}$. There are two choices for each of these terms coming from consecutive laps of $H$.

Suppose that $\left\{\tau_{k}\right\}_{k=0}^{m-1}=\min \left\{\left.\sigma^{i}\left(\theta_{1}\right)\right|_{m}, \ldots,\left.\sigma^{i}\left(\theta_{2^{n}}\right)\right|_{m}\right\}$. Let $\tau_{j}$ be the first term in $\left\{\tau_{k}\right\}_{k=0}^{m-1}$ that is allowed to be changed. Let $\tau_{j}^{*}$ denote the term with which $\tau_{j}$ is allowed to be replaced. Then it is clear that $\max \left\{\left.\sigma^{i}\left(\theta_{1}\right)\right|_{m}, \ldots,\left.\sigma^{i}\left(\theta_{2^{n}}\right)\right|_{m}\right\}$ must begin with $\tau_{1}, \ldots, \tau_{j-1}, \tau_{j}^{*}$.

Suppose that $\tau_{1}, \ldots, \tau_{j-1}, \tau_{j}$ has positive orientation, then by definition $\tau_{j+1}, \ldots$, $\tau_{2^{n}}$ is the smallest allowable string that can follow $\tau_{1}, \ldots, \tau_{j-1}, \tau_{j}$. Since $\tau_{j}$ and $\tau_{j}^{*}$ come from consecutive laps they have opposite orientations. Thus $\tau_{1}, \ldots, \tau_{j-1}, \tau_{j}^{*}$ has negative orientation, and so

$$
\max \left\{\left.\sigma^{i}\left(\theta_{1}\right)\right|_{m}, \ldots,\left.\sigma^{i}\left(\theta_{2^{n}}\right)\right|_{m}\right\}=\tau_{1}, \ldots, \tau_{j-1}, \tau_{j}^{*}, \tau_{j+1}, \ldots, \tau_{2^{n}} .
$$

A similar argument shows that when $\tau_{1}, \ldots, \tau_{j-1}, \tau_{j}$ has negative orientation we also have

$$
\max \left\{\left.\sigma^{i}\left(\theta_{1}\right)\right|_{m}, \ldots,\left.\sigma^{i}\left(\theta_{2^{n}}\right)\right|_{m}\right\}=\tau_{1}, \ldots, \tau_{j-1}, \tau_{j}^{*}, \tau_{j+1}, \ldots, \tau_{2^{n}} .
$$


Let $\alpha$ and $\theta_{i}$ be as stated in the above. Let $\alpha_{\min }^{i}=\min \left\{\left.\sigma^{i}\left(\theta_{1}\right)\right|_{m}, \ldots,\left.\sigma^{i}\left(\theta_{2^{n}}\right)\right|_{m}\right\}$ and analogously let $\alpha_{\max }^{i}=\max \left\{\left.\sigma^{i}\left(\theta_{1}\right)\right|_{m}, \ldots,\left.\sigma^{i}\left(\theta_{2^{n}}\right)\right|_{m}\right\}$. If $\alpha_{\min }^{i}$ has positive orientation then let $J_{i}$ denote the subinterval of $[0,1]$ defined by $J_{i}=\{x \in[0,1] \mid$ the $H$ itinerary of $\left.x \in\left[\left(\alpha_{\min }^{i}\right)^{\infty},\left(\alpha_{\max }^{i}\right)\left(\alpha_{\min }^{i}\right)^{\infty}\right]\right\}$. If $\alpha_{\min }^{i}$ has negative orientation then let $J_{i}$ denote the subinterval of $[0,1]$ defined by $J_{i}=\{x \in[0,1] \mid$ the $H$ itinerary of $\left.x \in\left[\left(\alpha_{\min }^{i}\right)\left(\alpha_{\max }^{i}\right)^{\infty},\left(\alpha_{\max }^{i}\right)^{\infty}\right]\right\}$. The following is immediate.

Lemma 10. If the itinerary $\tau$ is formed from any concatenation of terms from $\left\{\left.\sigma^{i}\left(\theta_{1}\right)\right|_{m}, \ldots,\left.\sigma^{i}\left(\theta_{2^{n}}\right)\right|_{m}\right\}$, then there exists $x \in J_{i}$ such that $\operatorname{It}(x)=\tau$ and $\operatorname{It}(H(x))$ consists of a concatenation of terms from $\left\{\left.\sigma^{i+1}\left(\theta_{1}\right)\right|_{m}, \ldots,\left.\sigma^{i+1}\left(\theta_{2^{n}}\right)\right|_{m}\right\}$, where $i+1$ is taken modulo $\mathrm{m}$.

Moreover, if It $(x)$ is periodic then the cycle corresponding to $x$ has a block structure over $\alpha$.

It should be noted that if $n=2$ then the concatenations correspond to *products. Thus we are, in some sense, generalizing $*$-products to the $n$-modal case. A slightly different approach to generalizing $*$-products is given in [6]. It is instructive to calculate the examples in [6] using the ideas in this section. (Llibre and Mumbru have also generalized $*$-products for certain special cases, see [8] for example.)

Proposition 11. Let $\beta$ be an n-modal cycle with a block structure over $\alpha$. Let $x$ be the leftmost point of a representative of $\beta$ in $H$. Then It $(x)$ can be formed by concatenating terms from $\left\{\left.\theta_{1}\right|_{m}, \ldots,\left.\theta_{2^{n}}\right|_{m}\right\}$.

Proof. Consider the "connect the dot" map for the portion of the orbit of $\beta$ given by $\beta^{l m}(1), \beta^{l m+1}(1), \ldots, \beta^{2 l m-1}(1)$. This has $n$ laps. If $\beta^{l m+i}(1)$ is not a critical point then the lap of $H$ in which $H^{l m+i}(x)$ lies is determined; if $\beta^{l m+i}(1)$ is a critical point then there are two possible choices for the lap in which $H^{l m+i}(x)$ lies. Referring back to the proof of Proposition 7, it is clear that if $\left\{\tau_{k}\right\}_{k=0}^{\infty}=I t(x)$ then $\left\{\tau_{k}\right\}_{k=l m}^{2 l m-1}=\left.\theta_{i}\right|_{m}$ for some i.

Example 12. The 2-modal cycle ( $\left.\begin{array}{llll}1 & 3 & 4 & 2\end{array}\right)$ has 4 representatives in the associated horseshoe map. These have itineraries $\left(\begin{array}{llll}1 & 2 & 3 & 2\end{array}\right)^{\infty},\left(\begin{array}{llll}1 & 2 & 3 & 1\end{array}\right)^{\infty},\left(\begin{array}{llll}1 & 3 & 3 & 1\end{array}\right)^{\infty}$ and $\left(\begin{array}{llll}1 & 3 & 3 & 2\end{array}\right)^{\infty}$. So if $x$ is the leftmost point of a representative of a cycle with block structure over $\left(\begin{array}{llll}1 & 3 & 4 & 2\end{array}\right)$ then $\operatorname{It}(x)$ can be expressed as a concatenation of terms from $\left\{\left(\begin{array}{llll}1 & 2 & 3 & 2\end{array}\right),\left(\begin{array}{llll}1 & 2 & 3 & 1\end{array}\right),\left(\begin{array}{llll}1 & 3 & 3 & 1\end{array}\right),\left(\begin{array}{llll}1 & 3 & 3 & 2\end{array}\right)\right\}$.

\section{The $2^{n}$ HORSESHOE OVER $\alpha$}

Let $H_{J}$ denote the truncation of $H$ defined in the following way. If $L_{\alpha}^{i}(1)$ is a critical point of $L_{\alpha}$, then truncate $H$ at $i /(n+1)$ so that the image of $J_{i}$ under this new map is $J_{i+1}$.

Lemma 13. For $i=0, \ldots, m-2$, we have $H_{J}\left(J_{i}\right)=J_{i+1}$ and $H_{J}\left(J_{m-1}\right)=J_{0}$.

Proof. The statement is trivially true if $L_{\alpha}^{i}(1)$ is a critical point of $L_{\alpha}$. These are the cases when $J_{i}$ contains points in two laps of $H_{J}$.

If $J_{i}$ is contained in a lap of $H_{J}$ then $H_{J}\left(J_{i}\right)$ consists of points that have itineraries contained in $\left\{\sigma(\tau) \mid \tau\right.$ is an itinerary of a point in $\left.J_{i}\right\}$. Since $H_{J}$ is monotone on $J_{i}$, it is enough to check that itineraries of the endpoints of $J_{i}$ are sent to the itineraries of the endpoints of $J_{i+1}$. 
Suppose that $i$ belongs to an increasing lap of $L_{\alpha}$. Then $J_{i}$ is contained in an increasing lap of $H_{J}$, and so it follows that

$$
\begin{gathered}
\sigma\left(\left(\alpha_{\text {max }}^{i}\right)^{\infty}\right)=\left(\alpha_{\text {max }}^{i+1}\right)^{\infty}, \quad \sigma\left(\left(\alpha_{\text {min }}^{i}\right)^{\infty}\right)=\left(\alpha_{\text {min }}^{i+1}\right)^{\infty}, \\
\sigma\left(\left(\alpha_{\text {min }}^{i}\right)\left(\alpha_{\text {max }}^{i}\right)^{\infty}\right)=\left(\alpha_{\text {min }}^{i+1}\right)\left(\alpha_{\text {max }}^{i+1}\right)^{\infty}, \\
\sigma\left(\left(\alpha_{\text {max }}^{i}\right)\left(\alpha_{\text {min }}^{i}\right)^{\infty}\right)=\left(\alpha_{\text {max }}^{i+1}\right)\left(\alpha_{\text {min }}^{i+1}\right)^{\infty} .
\end{gathered}
$$

Since $\sigma\left(\left(\alpha_{\text {min }}^{i}\right)^{\infty}\right)=\left(\alpha_{\text {min }}^{i+1}\right)^{\infty}$, both of $\alpha_{\text {min }}^{i}$ and $\alpha_{\text {min }}^{i+1}$ have the same orientation. So $H_{J}\left(J_{i}\right)=J_{i+1}$.

Suppose that $i$ belongs to a decreasing lap of $L_{\alpha}$. Then $J_{i}$ is contained in a decreasing lap of $H_{J}$, and so it follows that

$$
\begin{gathered}
\sigma\left(\left(\alpha_{\text {max }}^{i}\right)^{\infty}\right)=\left(\alpha_{\text {min }}^{i+1}\right)^{\infty}, \quad \sigma\left(\left(\alpha_{\text {min }}^{i}\right)^{\infty}\right)=\left(\alpha_{\text {max }}^{i+1}\right)^{\infty}, \\
\sigma\left(\left(\alpha_{\text {min }}^{i}\right)\left(\alpha_{\text {max }}^{i}\right)^{\infty}\right)=\left(\alpha_{\text {max }}^{i+1}\right)\left(\alpha_{\text {min }}^{i+1}\right)^{\infty}, \\
\sigma\left(\left(\alpha_{\text {max }}^{i}\right)\left(\alpha_{\text {min }}^{i}\right)^{\infty}\right)=\left(\alpha_{\text {min }}^{i+1}\right)\left(\alpha_{\text {max }}^{i+1}\right)^{\infty} .
\end{gathered}
$$

Since $\sigma\left(\left(\alpha_{\text {min }}^{i}\right)^{\infty}\right)=\left(\alpha_{\text {max }}^{i+1}\right)^{\infty}, \alpha_{\min }^{i}$ and $\alpha_{\max }^{i+1}$ have the same orientation and so $\alpha_{\min }^{i}$ and $\alpha_{\text {min }}^{i+1}$ have opposite orientations. So again we have $H_{J}\left(J_{i}\right)=J_{i+1}$.

The above argument holds if $i$ is replaced by $m-1$ and $i+1$ by 0 .

Proposition 14. The map $H_{J}^{m}$ restricted to $J_{i}$ consists of a $2^{n}$ horseshoe.

Proof. Lemma 13 shows that $H_{J}^{m}$ maps $J_{i}$ onto itself. For $k=0, \ldots, m-1$ we know that exactly $n$ of the intervals $J_{k}$ contain two laps and the others contain one lap. Thus it follows from Lemma 13 that $H_{J}^{m}$ restricted to $J_{i}$ can have at most $2^{n}$ laps. From Lemma 10 and the construction of $H_{J}$ it is clear that $H_{J}^{m}$, restricted to $J_{i}$, has laps labelled by $\left\{\left.\sigma^{i}\left(\theta_{1}\right)\right|_{m}, \ldots,\left.\sigma^{i}\left(\theta_{2^{n}}\right)\right|_{m}\right\}$. Lemma 9 shows that these truncated itineraries, and thus the laps, are distinct. So $H_{J}^{m}$ restricted to $J_{i}$ has exactly $2^{n}$ laps.

From Lemma 10 and the construction of $H_{J}$ it is clear that the itineraries of points in $J_{i}$ form the full $n$-shift on the set $\left\{\left.\sigma^{i}\left(\theta_{1}\right)\right|_{m}, \ldots,\left.\sigma^{i}\left(\theta_{2^{n}}\right)\right|_{m}\right\}$. Thus $H_{J}^{m}$ restricted to $J_{i}$ consists of the full $2^{n}$ horseshoe.

The above proof with Lemma 10 and Proposition 11 give us the following theorem.

Theorem 15. Let $\alpha, \beta$ and $\gamma$ be n-modal cycles. If $\beta$ has a block structure over $\alpha$, then $\beta$ forces $\alpha$ and $\beta$ has representatives in $H_{J}$.

If $\gamma$ forces $\alpha$ and $\gamma$ has a representative in $H_{J}$, then $\gamma$ has a block structure over $\alpha$.

Example 16. Suppose $\alpha=\left(\begin{array}{llll}1 & 3 & 4 & 2\end{array}\right)$. Then $H_{J}^{4}$ restricted to $J_{1}$ is a 4 -horseshoe. The laps are labelled from left to right by $1332,1331,1231$ and 1232 . Notice that 1332 has negative orientation and so the first lap of the horseshoe is decreasing. Let $\beta=\left(\begin{array}{llllll}1 & 3 & 6 & 4 & 5 & 2\end{array}\right)$. Notice that $\beta$ is 3 -modal, and so representatives of $\beta$ can be found in the horseshoe. For example, the itinerary of one such representative

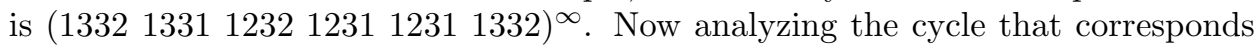
to the point in $H_{J}$ with itinerary $(133213311232123112311332)^{\infty}$ gives the cycle of type $\alpha^{\beta}$. 
Notice that different choices of representatives for $\beta$ in the horseshoe can give different cycles that have type $\alpha^{\beta}$. Two such cycles for the above values of $\alpha$ and $\beta$ are the cycles

(118201131624761319124152385142110217229)

and

(1 182011316247613191241523851422921721 10).

A basic result on the forcing relation on the set of cycles with block structure over $\alpha$ is the following.

Theorem 17. Suppose $\gamma$ is an n-modal cycle of type $\alpha^{\beta}$. Then for any cycle $\eta$ such that $\beta$ forces $\eta$ there is a cycle $\delta$ of type $\alpha^{\eta}$ such that $\gamma$ forces $\delta$.

Proof. Let $P$ denote a representative of $\gamma$ in $H$. Form the truncated horseshoe map $H_{P}$. Then for some $i, H_{P}^{n}$ restricted to $J_{i}$ has a representative of $\beta$. Since $\beta$ forces $\eta, H_{P}^{n}$ restricted to $J_{i}$ has a representative of $\eta$. Thus $H_{P}$ must have a representative of cycle type $\alpha^{\eta}$. Theorem 2 completes the proof.

\section{Cycles without Block Structure that Force $\alpha$}

It will be shown that if $\beta$ forces $\alpha$ and $\beta$ does not have a block structure over $\alpha$, then $\beta$ must force a full 2-horseshoe of simple extensions of $\alpha$.

The theorem below is related to a theorem of Misiurewicz and Nitecki ([10], Theorem 9.12). In [10] the idea of positive forcing is introduced. The authors show that if $\beta$ positively forces $\alpha$ and $\beta$ does not have a block structure over $\alpha$, then $\beta$ must force a full 2-horseshoe of simple extensions of $\alpha$.

Theorem 18. Let $\alpha$ and $\beta$ be n-modal cycles. Suppose that $\beta$ forces $\alpha$ and that $\beta$ does not have a block structure over $\alpha$. Then for any unimodal cycle $\gamma, \beta$ forces a simple extension of $\alpha$ by $\gamma$.

Proof. Let $Q$ denote a representative of $\beta$ in $H$. Let $H_{Q}$ denote the associated truncated horseshoe map. Since $\beta$ does not have a block structure over $\alpha$, it follows that for each $J_{i}$ there must be a $k$ such that $H_{Q}^{k}\left(J_{i}\right) \nsubseteq J_{i+k}$. If $k$ is the smallest positive integer with this property, draw an arrow from $J_{i}$ to $J_{i+k}$. Observe that $H_{Q}^{k}\left(J_{i}\right) \supset J_{i+k}$.

Since each vertex $J_{i}$ has an arrow leaving it, and since there are only a finite number of vertices, there must be a loop containing $J_{i_{1}}, \ldots, J_{i_{l}}$ and integers $m_{1}, \ldots, m_{l}$ such that $H_{Q}^{m_{1}}\left(J_{i_{1}}\right) \supset J_{i_{2}}, \ldots, H_{Q}^{m_{l}}\left(J_{i_{l}}\right) \supset J_{i_{1}}$.

Choose the loop to be shortest possible and choose the labelling such that $i_{1}<i_{k}$ for $k=2, \ldots, l$. Then notice that $i_{k-1}$ cannot be greater than $i_{k}$, because if it was, the fact that $H_{Q}^{i_{k-1}-i_{k}}\left(J_{i_{k}}\right) \subseteq J_{i_{(k-1)}}$ would imply that there is an arrow from $J_{i_{k-1}}$ to $J_{i_{k+1}}$, and so there would be a shorter loop. Thus $i_{1}<i_{2}<\cdots<i_{l}$ and, for each $k, m_{k}=i_{k+1}-i_{k}$. Therefore, for each $k$ we have $H_{Q}^{m}\left(J_{i_{k}}\right) \supset J_{i_{k}}$.

If at least one of the $J_{i_{k}}$ contains a maximum or a minimum, choose one of these intervals and denote it by $J_{C}$. If $H_{Q}$ is monotonic on all of the $J_{i_{k}}$, find the first $r$ such that $J_{i_{1}+r}$ is not monotonic and denote it by $J_{C}$. Notice that in either case $H_{Q}^{m}\left(J_{C}\right) \supset J_{C}$.

Then it is clear from the construction of $H_{Q}$ that there exist closed intervals $I_{i}$ in $J_{i}$ such that $H_{Q}\left(I_{C-1}\right)=J_{C}, H_{Q}\left(I_{C-2}\right)=I_{C-1}, H_{Q}\left(I_{C-3}\right)=I_{C-2}, \ldots, H_{Q}\left(I_{C+1}\right)$ 
$=I_{C+2}$, (where the subscripts are taken modulo $m$ ) and such that $H_{Q}$ is monotonic on $I_{i}$.

Again from the construction of $H_{Q}$, there exist two closed subintervals $I_{C_{1}}$ and $I_{C_{2}}$ of $J_{C}$ such that $H_{Q}$ is monotone increasing on one of these intervals and monotone decreasing on the other, and such that $H_{Q}\left(I_{C_{1}}\right)=H_{Q}\left(I_{C_{2}}\right)=I_{C+1}$. Then $H_{Q}^{m}\left(I_{C_{1}}\right) \supset I_{C_{1}} \cup I_{C_{2}}$ and $H_{Q}^{m}\left(I_{C_{2}}\right) \supset I_{C_{1}} \cup I_{C_{2}}$. So under $H_{Q}^{m}$ we have the full 2-horseshoe on intervals $I_{C_{1}}$ and $I_{C_{2}}$. Each of the cycles given by this horseshoe is simple because $H_{Q}$ is monotonic on the intervals $I_{i}$.

\section{REFERENCES}

[1] Ll. Alsedà, J. Llibre, and M. Misiurewicz, Combinatorial dynamics and entropy in dimension one, World Scientific, River Edge, NJ, 1993. MR 95j:58042

[2] S. Baldwin, Generalizations of a theorem of Sarkovskii on orbits of continuous real-valued functions, Discrete Math. 67 (1987), 111-127. MR 89c:58057

[3] C. Bernhardt, E. Coven, M. Misiurewicz, I. Mulvey, Comparing periodic orbits of maps of the interval, Trans. Amer. Math. Soc. 333 (1992), 701-707. MR 92m:58106

[4] C. Bernhardt, The ordering of permutations induced by continuous maps of the real line, Ergodic Theory Dynamical Systems 7 (1987), 155-160. MR 88h:58099

[5] C. Bernhardt, Self-similarity maps for the set of unimodal cycles, Proc. Conf. "Thirty Years After Sarkovskiı̌'s Theorem: New Perspectives" (Murcia, 1994), Internat. J. Bifurcation Chaos Appl. Sci. Ergrg. 5 (1995), 1325-1330. CMP 1996:4

[6] K. Brucks, R. Galeeva, D. Rockmore and C. Tresser, On the *-product in kneading theory, Preprint.

[7] P. Collet and J-P. Eckmann, Iterated maps on the interval as dynamical systems, Progress in Phys., vol. 1, Birkhäuser, Boston, 1980. MR 82j:58078

[8] J. Llibre and P. Mumbru, Extending the *-product, European Conf. Iteration Theory (Batschuns, 1989), World Scientific Publ., Singapore, 1991, pp. 199-214. MR 93f:58125

[9] J. Milnor and W. Thurston, On iterated maps of the interval, Dynamical Systems (College Park, MD, 1986/87), Lecture Notes in Math 1342 (1988), 465-563. MR 90a:58083

[10] M. Misiurewicz and Z. Nitecki, Combinatorial patterns for maps of the interval, Memoirs of AMS 94 (456) (1991). MR 92h:58105

Department of Mathematics and Computer Science, Fairfield University, Fairfield Connecticut 06430

E-mail address: cbernhardt@fair1.fairfield.edu 